\title{
Identification of leaf volatiles from olive (Olea europaea) and their possible role in the ovipositional preferences of olive fly, Bactrocera oleae (Rossi) (Diptera: Tephritidae)
}

\author{
Ricardo Malheiro $^{\mathrm{a}, \mathrm{b}}$, Susana Casal ${ }^{\mathrm{b}, *}$, Sara C. Cunha ${ }^{\mathrm{b}}$, Paula Baptista ${ }^{\mathrm{a}}$, José Alberto Pereira ${ }^{\mathrm{a}, *}$ \\ ${ }^{a}$ Mountain Research Centre (CIMO), School of Agriculture, Polytechnic Institute of Bragança, Campus de Santa Apolónia, Apartado 1172, 5301-855 Bragança, Portugal \\ ${ }^{\mathrm{b}}$ REQUIMTE/Laboratory of Bromatology and Hydrology, Faculty of Pharmacy, Porto University, Rua de Jorge Viterbo Ferreira, 228, 4050-313 Porto, Portugal
}

\section{A R T I C L E I N F O}

\section{Article history:}

Received 26 May 2015

Received in revised form 8 October 2015

Accepted 16 October 2015

Available online 22 October 2015

\section{Keywords:}

Olea europaea L. (Oleaceae)

Olive fly

Bactrocera oleae (Tephritidae)

Olive leaf

Volatiles

Olive cultivar

Oviposition preference

\begin{abstract}
A B S T R A C T
The olive fly, Bactrocera oleae (Rossi), is a monophagous pest that displays an oviposition preference among cultivars of olive (Olea europaea L.). To clarify the oviposition preference, the olive leaf volatiles of three olive cultivars (Cobrançosa, Madural and Verdeal Transmontana) were assessed by headspace solid-phase microextraction gas chromatography-mass spectrometry (HS-SPME-GC/MS) at six different periods of olive fruit maturation and degrees of infestation.

A total of 39 volatiles were identified, mainly esters and alcohols, with a minor percentage of aldehydes, ketones and terpenic compounds, including sesquiterpenes. At sampling dates with higher degrees of infestation, cv. Cobrançosa had, simultaneously, significantly lower infestation degrees and higher volatile amounts than the other two cultivars, with a probable deterrent effect for oviposition. The green leaf volatiles (GLVs) (Z)-3-hexen-1-ol and ( $Z$ )-3-hexen-1-ol acetate) were the main compounds identified in all cultivars, together with toluene. The abundance of GLVs decreased significantly throughout maturation, without significant differences among cultivars, while toluene showed a general increase and positive correlation with olive fly infestation levels.

The results obtained could broaden our understanding of the roles of various types and amounts of olive volatiles in the environment, especially in olive fly host selection and cultivar preference.
\end{abstract}

(c) 2015 Elsevier Ltd. All rights reserved.

\section{Introduction}

Plant volatiles are ascribed various important roles and functions as the means by which plants communicate and interact with the surrounding environment. Volatiles participate in plant reproduction, in tritrophic interactions, in belowground defense systems and in the abiotic stress response of plants (Dudareva et al., 2006). Regarding defense mechanisms, plants release volatile compounds with deterrent and repellent purposes to keep herbivores and pests away or, when attacked, to attract specific pest predators, parasitoids and other natural enemies and alert neighboring plants (Tamiru et al., 2011; Unsicker et al., 2009; Wu and Baldwin, 2010). Such compounds are globally recognized as semiochemicals (Paré and Tummlinson, 1999).

Several pests and diseases attack olive (Olea europaea L.) trees each year, causing serious production losses. Bactrocera oleae (Rossi) (Diptera: Tephritidae), the olive fly, is considered the key

\footnotetext{
* Corresponding authors.

E-mail addresses: sucasal@ff.up.pt (S. Casal), jpereira@ipb.pt (J.A. Pereira).
}

pest in several regions in the world, particularly in the Mediterranean area (Daane and Jonhson, 2010), causing important economic and quality losses (Malheiro et al., 2015a). The female of this dipteran shows cultivar preference by attacking specific olive cultivars (Burrack and Zalom, 2008; Iannotta et al., 2007; Navrozidis et al., 2007; Gonçalves et al., 2012). Host selection for oviposition is believed to be based on chemical (Imperato et al., 2012; Kombargi et al., 1998; Spadafora et al., 2008), physical (Neuenschwander et al., 1985; Rizzo et al., 2012), and molecular aspects (Corrado et al., 2012). More recently, increasing evidence has been found that microbiological factors are also important in the oviposition preference of the olive fly. Indeed, fungi and bacteria are also believed to be involved in the complex relationship between the olive tree and the olive fly (Liscia et al., 2013), in addition to epiphytic communities that may be related to adult olive fly survival (Sacchetti et al., 2008).

Regarding chemical factors, olive tree volatiles (fruits and leaf) may play an important role in olive fly cultivar selection (Aluja and Mangan, 2008; Malheiro et al., 2015b). It was recently shown that when olives are attacked by olive fly females, phytohormones, 
volatiles and defense proteins are released, as reported by Alagna et al. (2015). However, most studies on cultivar preference are mainly focused on olive fruits (Malheiro et al., 2015b), rather than olive leaves or the olive tree as a whole. Therefore, studies on olive leaf volatile characterization are scarce, and most are based on hydro-distillate volatile fractions, which are different from the natural emissions (Brahmi et al., 2012; Campeol et al., 2001, 2003; Flamini et al., 2003). Nevertheless, some studies do report the potential effect of olive leaf volatiles in olive fly behavior, and according to Scarpati et al. (1993) two abundant olive leaf volatiles, toluene and $\alpha$-pinene, were the most attractive and repellent cues in olive fly oviposition bioassays, respectively. However, Liscia et al. (2013), in studying the electrophysiological response of females (mated and unmated) to volatiles from olive leaves and fruits, did not find significant differences. These apparently contradictory results suggest that the host-pest interaction, $O$. europaea-B. oleae, is complex and difficult to study, and therefore it is not yet well understood. A detailed knowledge of the volatile patterns produced by olive leaves from cultivars with different degrees of susceptibility to olive fly attack would be very useful to clarify this interaction. Furthermore, olive leaves represent a major part of the olive tree and may decisively influence olive fly oviposition preference, not only by chemical but also by physical cues. Therefore, in this work, the volatile compounds emitted by olive leaves from cvs. Cobrançosa, Madural and Verdeal Transmontana were assessed at different harvest times during fruit maturation. These olive cultivars were selected due to their different susceptibilities to the olive fly in Portugal, where the Verdeal Transmontana and Madural cultivars are highly susceptible, while cv. Cobrançosa is less attacked (Gonçalves et al., 2012). The possible relationships between volatile types and amounts and cultivar susceptibility to $B$. oleae attack are discussed as well. To the authors' knowledge, this investigation is the first to relate olive fly oviposition preference to the volatiles from olive leaves from different cultivars.

\section{Results}

\subsection{Infestation level and maturation index}

Fruit infestation levels in cvs. Cobrançosa, Madural and Verdeal Transmontana were assessed fortnightly to observe the preferences of the olive fly towards the three olive cultivars during fruit maturation. The results obtained are reported in Fig. 1. During the assessed period (from 4th Aug. to 23rd Nov.), higher infestation
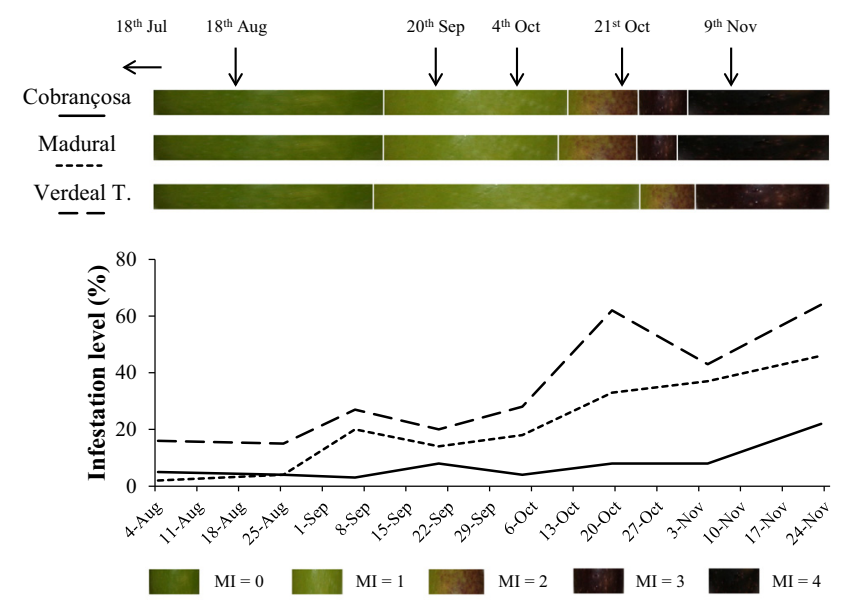

Fig. 1. Bactrocera oleae infestation levels (\%) and fruits maturation from the cultivars Cobrançosa, Madural and Verdeal Transmontana. levels were clearly observed in cv. Verdeal Transmontana, followed by cv. Madural, and finally, cv. Cobrançosa was the least attacked olive cultivar. During August, low infestation levels were observed, with $16 \%$ in cv. Verdeal Transmontana, while cvs. Madural and Cobrançosa had 5 and 2\% infestation, respectively. During this period, the olive maturation index (MI) was 0 for all cultivars (Fig. 1). In September, the infestation levels increased in cvs. Madural and Verdeal Transmontana, while cv. Cobrançosa maintained a low infestation rate. In this period, all cultivars reported MI between 0 and 1 (Fig. 1). In October, a considerable increase in infestation was observed in olive fruits of cvs. Verdeal Transmontana and Madural, reaching 62 and 33\%, respectively, on 19th Oct, while cv. Cobrançosa had only $8 \%$ infested fruits. At the end of October, olive fruits from cvs. Cobrançosa and Madural showed a MI of approximately 3, while cv. Verdeal Transmontana showed a MI between 1 and 2 (Fig. 1). Near the olive harvest (23rd Nov), the infestation levels in cvs. Verdeal Transmontana and Madural were 64 and 46\%, respectively, while in Cobrançosa the level was approximately $22 \%$. At that date, olives from Cobrançosa and Madural had already reached advanced maturation (MI between 4 and 5), while cv. Verdeal Transmontana showed a slower maturation process, as olives from this cultivar showed a MI of 3 (Fig. 1), which is a natural characteristic of this cultivar. No significant differences were observed in the infestation levels in the three olive cultivars until approximately the first week of October $(P>0.05)$. From that moment, significant differences were observed among the three cultivars, with olives from cv. Verdeal Transmontana exhibiting significantly higher infestation levels than olives from cv. Cobrançosa ( $P=0.013$ and $P=0.010$ for 21 st Oct. and 9th Nov.).

\subsection{Volatile amounts and composition}

The volatile fraction of olive leaves from cvs. Cobrançosa, Madural, and Verdeal Transmontana were assessed at six different harvest periods during olive fruit maturation. Overall, 39 volatile compounds were identified: 5 alcohols, 3 aldehydes, 12 esters, 3 ketones, 9 sesquiterpenes, 4 terpenes, and 3 aromatic hydrocarbons. Quantitative and qualitative changes were observed among olive cultivar and harvest period. Considerable quantitative variations were also observed in the volatile composition of olive leaves according to the collection date and olive cultivar assessed. In Fig. 2, higher amounts of volatile emissions are observed for cv. Cobrançosa leaves, followed by cvs. Madural and Verdeal Transmontana. As of 18th Jul (first sampling date), no significant differences $(P=0.325)$ were observed among the three olive cultivars, with the total volatile emission varying between

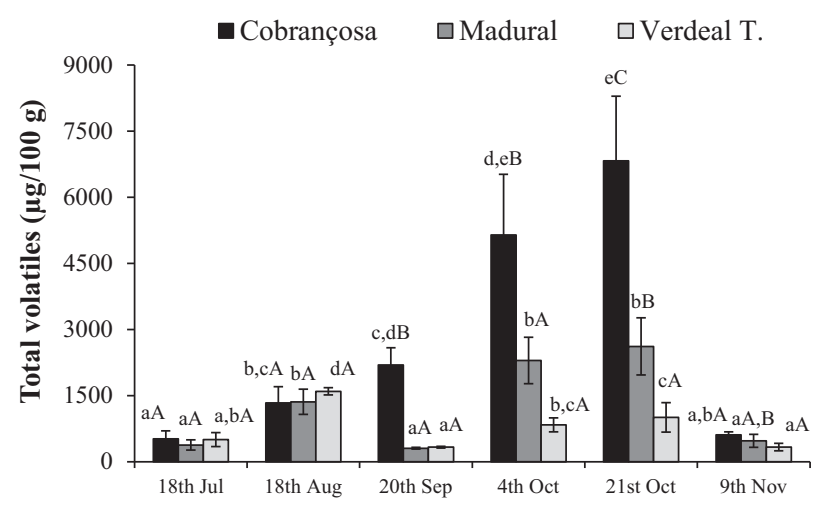

Fig. 2. Total volatiles emission ( $\mu \mathrm{g} / 100 \mathrm{~g}$ of leaf) of cvs. Cobrançosa, Madural and Verdeal Transmontana olive leaf at different harvesting times during fruit maturation (in each cultivar different minor letters represent significant differences during crop maturation $(P<0.05)$; in each harvest moment, capital letters represent significant differences between olive cultivars $(P<0.005)$. 
$379 \mu \mathrm{g} / 100 \mathrm{~g}$ leaf (cv. Madural) and $519 \mu \mathrm{g} / 100 \mathrm{~g}$ (cv. Cobrançosa). One month later, total volatile emission had almost tripled in all three cultivars, also without significant differences among the studied cultivars $(P=0.280)$. At the subsequent harvest times, high volatile emission was consistently recorded in cv. Cobrançosa compared to the other two olive cultivars: 2194, 5143, and $6830 \mu \mathrm{g} / 100 \mathrm{~g}$, respectively, at 20th Sep, 4th Oct, and 21st Oct $(P<0.001$ for the three assessed harvest moments). At the last sampling date, volatile emissions from the three olive cultivars dropped considerably to values from $332 \mu \mathrm{g} / 100 \mathrm{~g}$ (cv. Verdeal Transmontana) to $608 \mu \mathrm{g} / 100 \mathrm{~g}$ (cv. Cobrançosa), still showing significant differences among cultivars $(P=0.006)$.

Globally, 36 compounds were identified in cv. Cobrançosa (Table 1), 28 in cv. Madural (Table 2), and 30 in cv. Verdeal Transmontana (Table 3).

Independently of the olive cultivar, characteristic GLVs were the predominant volatiles present in olive leaves. Esters were the main chemical class identified in all cultivars analyzed (Fig. 3) during the different sampling dates assessed. As of the first sampling date, their content varied between $83.9 \%$ in cv. Verdeal Transmontana and $95.6 \%$ in cv. Madural (Fig. 2). Throughout the study, esters decreased in relative abundance, with small variations in cv. Verdeal Transmontana between the second (18th Aug) and last sampling dates (9th Nov). In cv. Cobrançosa, a considerable increase was observed from the fifth to the sixth and last sampling date (Fig. 3). Such trends were dependent on the individual esters identified in the volatile fraction of the olive leaves. For instance, $(Z)-3-$ hexen-1-ol acetate was the most abundant compound among all the volatiles identified in the three cultivars. This ester decreased significantly during the surveyed period, especially in cv. Verdeal Transmontana $(P=0.013)$. In $\mathrm{cv}$. Cobrançosa, it increased from the 18th Jul to 20th Sep and afterwards decrease until the end of the surveyed period (9th Nov), reaching a minimum value of $32.4 \%$ (Table 1). By contrast, in cv. Madural, the same compound decreased from 18th Jul to 20th Sep and afterwards increased to maximum value of $52.3 \%$ at 21 st Oct. From this date until the end, $(Z)$-3-hexen-1-ol acetate decreased (Table 2). For cv. Verdeal Transmontana, the reduction was more evident, from $50.4 \%$ to $10.4 \%$ (first and last dates; Table 3). Another ester, the methyl ester of 2-methyl-butanoic acid, was present in relatively high abundance at the first sampling date with 14.9, 26.5, and 13.6\% in cvs. Cobrançosa, Madural and Verdeal Transmontana, respectively. This compound also decreased significantly $(P=0.002$ for cvs. Cobrançosa and Madural; $P=0.005$ for cv. Verdeal Transmontana) throughout fruit maturation. In cv. Madural, it decreased until 4 th Oct with a minimum value of $4.0 \%$, then increased until 9th Nov (11.8\%; Table 2). In cv. Verdeal Transmontana, the maximum values were reported as of 18 th Jul (13.6\%), decreasing significantly until 18th Aug (2.5\%), then increasing again to $20 \mathrm{Sep}$ (8.3\%), and decreasing steadily afterwards until 9th Nov (3.4\%). The methyl ester of 3-methyl-butanoic acid showed an opposite trend. This compound increased considerably during fruit maturation. In cV. Cobrançosa, it varied between 6.1 and $2.2 \%$ from 18 th Jul to 21 st Oct, with the minimum abundance on 4 th Oct (0.52\%). A significant maximum value was quantified as of 9 th Nov with $39.3 \%$. A similar trend was observed in cv. Verdeal Transmontana, with significantly higher abundance as of 9th Nov (33.6\%). In this cultivar, butanoic acid, 3-methyl-, methyl ester showed values ranging from 5.4 to $1.7 \%$ between 18 th Jul and 21 st Oct, with minimum values on 18th Aug (0.9\%). In cv. Madural, butanoic acid, 3-methyl-, methyl ester was absent from the third sampling (20th Sep), and higher values were recorded on 9th Nov, at $9.0 \%$, although this value was significantly lower than in cvs. Cobrançosa and Verdeal Transmontana assessed during the same period. From the remaining esters identified, the significantly higher abundance of butanoic acid methyl ester in cv. Madural on 4th Oct, at $21.2 \%$, should be highlighted (Table 2).

Regarding alcohols, five compounds were identified, namely 3-methyl-1-butanol, 3-hexanol, (Z)-3-hexen-1-ol, 1-hexanol and 1-octanol (Table 1-3). All five compounds occurred in cvs. Cobrançosa and Verdeal Transmontana, while in cv. Madural, only 3-methyl-1-butanol, 1-hexanol, and (Z)-3-hexen-1-ol were detected. Nevertheless, only (Z)-3-hexen-1-ol was present throughout the entire surveyed period in all cultivars, being the main alcohol identified. During the surveyed period, a similar trend of alcohol production was observed in the three cultivars. (Z)-3-hexen-1-ol and total alcohol abundance generally increased from 18th Jul to 21st Oct (Table 1-3, and Fig. 3) and afterwards decreased until 9th Nov. In cv. Cobrançosa, minimum values of (Z)-3-hexen-1-ol were detected on 18th Aug (1.8\%), in cv. Madural on 18th Jul (1.3\%), and in cv. Verdeal Transmontana as of 20th Sep (5.0\%). Maximum values of $(Z)$-3-hexen-1-ol were reported as of 21 st Oct in the three cultivars in the following increasing order: cv. Madural (12.53\%; Table 2) <cv. Verdeal Transmontana (20.6\%; Table 3) $<$ cv. Cobrançosa (27.4\%; Table 1). Regarding total alcohol abundance, an additional peak production in cv. Verdeal Transmontana must be highlighted, as of 18th Aug, with a value of $37.45 \%$ (Fig. 1). This increase is related to the high abundance of 3-methyl-1-butanol (15.1\%; Table 3 ), while in the remaining cultivars, this alcohol consistently occurred at values below $1 \%$.

Aldehydes were present in low amounts. The identified aldehydes were hexanal, nonanal and decanal. These aldehydes were present in cvs. Cobrançosa and Madural, but nonanal was absent in cv. Verdeal Transmontana (Table 3). The maximum percentage in aldehydes was reported as of the second date (18th Aug) in all cultivars, with a maximum of $1.0 \%$ in cv. Cobrançosa. Afterward, aldehydes decreased and became undetectable in the volatile fraction of olive leaves from the three cultivars (Fig. 3).

Three ketones were identified: 3-pentanone, 3-hexanone, and 6-methyl-5-hepten-2-one. Ketones were not found in cv. Cobrançosa, and in cv. Madural only 6-methyl-5-hepten-2-one was identified as of 18 th Aug with a low percentage, $0.7 \%$ (Table 2). In cv. Verdeal Transmontana, ketones were only identified on the first two surveyed dates and were responsible for approximately $6.5 \%$ of the volatile fraction as of 18th Aug (Fig. 1), particularly 3-pentanone, which was exclusively found in this olive cultivar.

Sesquiterpenes are a minority fraction of the volatile composition of olive leaves from the cultivars studied. The relative percentages of sesquiterpenes varied between $0.2 \%$ and $1.5 \%$ (Fig. 1 ), with $\beta$-caryophyllene as the most representative one (for detailed sesquiterpene composition consult Table 1-3). Significantly higher $\beta$-caryophyllene content $(P=0.003)$ was reported as of 18 th Jul in cv. Verdeal Transmontana (1.1\%), dropping abruptly to $0.1 \%$ as of 18th Aug and showing slight increases thereafter until $0.4 \%$ at 9th Nov (Table 3).

Terpenes, namely monoterpenic compounds, exhibited a similar trend in cvs. Cobrançosa and Madural. For these cultivars, higher contents of terpenes were reported as of 18th Aug and 9 th Nov (second and last dates, respectively; Fig. 1). In the case of cv. Verdeal Transmontana, higher terpene abundance was reported as of 20th Sep and 9th Nov. Limonene was consistently the most abundant monoterpene, with higher values observed in cvs. Cobrançosa and Madural on 18th Aug and 9th Nov, while in cv. Verdeal Transmontana, it represented $8 \%$ of the volatile fraction on 9th Nov, significantly higher than in the remaining cultivars $(P=0.007)$. $\alpha$-Pinene was identified in all three cultivars but only on the third date (20th Sept), ranging between 0.1 and $0.3 \%$. $\rho$-Cymene was not identified in the volatile profile of $\mathrm{cv}$. Verdeal Transmontana but was present in cvs. Cobrançosa and Madural (Table $1-3)$ in low amounts $(\leqslant 0.5 \%)$. 
Table 1

Volatile composition (\%; mean \pm standard error) of cv. Cobrançosa olive leaf at different harvest times.

\begin{tabular}{|c|c|c|c|c|c|c|c|c|}
\hline Chemical class & Compound & 18th Jul & 18th Aug & 20th Sep & 4th Oct & 21st Oct & 9th Nov & $P$-value \\
\hline Alcohols & $\begin{array}{l}\text { 3-Methyl-1-butanol } \\
\text { 3-Hexanol } \\
\text { (Z)-3-hexen-1-ol } \\
\text { Hexanol } \\
\text { Octanol }\end{array}$ & $\begin{array}{l}0.5 \pm 0.2 \mathrm{a} \\
- \\
4.0 \pm 1.7 \mathrm{a}, \mathrm{b} \\
- \\
-\end{array}$ & $\begin{array}{l}0.3 \pm 0.0 \mathrm{a} \\
1.3 \pm 0.5 \mathrm{a} \\
1.8 \pm 0.5 \mathrm{a} \\
- \\
-\end{array}$ & $\begin{array}{l}0.4 \pm 0.2 \mathrm{a} \\
0.1 \pm 0.0 \mathrm{a} \\
4.3 \pm 1.1 \mathrm{a}, \mathrm{b} \\
- \\
-\end{array}$ & $\begin{array}{l}\text { tr. } \\
\text { tr. } \\
12.6 \pm 1.3 \mathrm{c} \\
- \\
-\end{array}$ & $\begin{array}{l}0.2 \pm 0.0 \mathrm{a} \\
- \\
27.4 \pm 2.1 \mathrm{~d} \\
1.4 \pm 0.3 \\
\text { tr. }\end{array}$ & $\begin{array}{l}- \\
- \\
9.8 \pm 2.7 \mathrm{~b}, \mathrm{c} \\
- \\
-\end{array}$ & $\begin{array}{l}0.172^{1} \\
0.094^{1} \\
<0.001^{2} \\
- \\
-\end{array}$ \\
\hline Aldehydes & $\begin{array}{l}\text { Hexanal } \\
\text { Nonanal } \\
\text { Decanal }\end{array}$ & $\begin{array}{l}0.8 \pm 0.3 \mathrm{~b} \\
- \\
-\end{array}$ & $\begin{array}{l}- \\
0.5 \pm 0.2 \mathrm{~b} \\
0.6 \pm 0.1\end{array}$ & $\begin{array}{l}0.1 \pm 0.0 \mathrm{a} \\
0.1 \pm 0.0 \mathrm{a} \\
-\end{array}$ & $\begin{array}{l}- \\
\operatorname{tr} \\
\text { tr. }\end{array}$ & $\begin{array}{l}\text { tr. } \\
\text { tr. } \\
-\end{array}$ & $\begin{array}{l}- \\
- \\
-\end{array}$ & $\begin{array}{l}0.030^{2} \\
0.044^{2} \\
-\end{array}$ \\
\hline Esters & $\begin{array}{l}\text { Butanoic acid methyl ester } \\
\text { Butanoic acid, 3-methyl-, methyl ester } \\
\text { Butanoic acid, 2-methyl-, methyl ester } \\
\text { Hexanoic acid methyl ester } \\
\text { (Z)-3-Hexenoic acid methyl ester } \\
\text { (E)-2-Hexenoic acid methyl ester } \\
\text { (Z)-3-Hexen-1-ol acetate } \\
\text { Hexyl acetate } \\
\text { Benzoic acid methyl ester } \\
\text { (Z)-3-Hexenyl isobutyrate } \\
\text { (Z)-3-Hexenyl butyrate } \\
\text { (E)-3-Hexenyl ester }\end{array}$ & $\begin{array}{l}8.2 \pm 2.6 \mathrm{~b} \\
6.1 \pm 2.8 \mathrm{a} \\
14.9 \pm 2.1 \mathrm{c} \\
0.7 \pm 0.1 \mathrm{a}, \mathrm{b} \\
4.3 \pm 1.9 \mathrm{a} \\
- \\
49.8 \pm 6.6 \mathrm{a}-\mathrm{c} \\
1.0 \pm 0.3 \mathrm{a} \\
2.2 \pm 1.1 \mathrm{~b} \\
- \\
- \\
2.4 \pm 1.0\end{array}$ & $\begin{array}{l}8.4 \pm 1.6 \mathrm{~b} \\
1.3 \pm 0.5 \mathrm{a} \\
10.3 \pm 3.4 \mathrm{~b}, \mathrm{c} \\
1.0 \pm 0.3 \mathrm{a}, \mathrm{b} \\
- \\
- \\
58.9 \pm 8.7 \mathrm{~b}, \mathrm{c} \\
1.4 \pm 0.3 \mathrm{a} \\
2.4 \pm 0.6 \mathrm{~b} \\
- \\
- \\
-\end{array}$ & $\begin{array}{l}3.7 \pm 1.5 \mathrm{a} \\
5.6 \pm 1.9 \mathrm{a} \\
1.4 \pm 0.7 \mathrm{a} \\
0.4 \pm 0.0 \mathrm{a} \\
2.7 \pm 0.9 \mathrm{a} \\
- \\
68.7 \pm 2.0 \mathrm{c} \\
0.7 \pm 0.1 \mathrm{a} \\
0.4 \pm 0.1 \mathrm{a} \\
- \\
1.1 \pm 0.2 \mathrm{a} \\
-\end{array}$ & $\begin{array}{l}8.9 \pm 2.8 \mathrm{~b} \\
0.5 \pm 0.1 \mathrm{a} \\
3.3 \pm 0.7 \mathrm{a}, \mathrm{b} \\
1.0 \pm 0.1 \mathrm{a}, \mathrm{b} \\
4.5 \pm 0.8 \mathrm{a} \\
- \\
57.6 \pm 4.2 \mathrm{~b}, \mathrm{c} \\
0.5 \pm 0.1 \mathrm{a} \\
2.6 \pm 0.7 \mathrm{~b} \\
0.2 \pm 0.0 \\
3.3 \pm 0.3 \mathrm{~b} \\
-\end{array}$ & $\begin{array}{l}2.4 \pm 0.6 \mathrm{a} \\
2.2 \pm 0.4 \mathrm{a} \\
0.6 \pm 0.2 \mathrm{a} \\
1.7 \pm 0.5 \mathrm{~b} \\
11.8 \pm 1.5 \mathrm{~b} \\
1.0 \pm 0.2 \\
42.6 \pm 4.1 \mathrm{a}, \mathrm{b} \\
1.1 \pm 0.2 \mathrm{a} \\
0.5 \pm 0.1 \mathrm{a} \\
- \\
- \\
-\end{array}$ & $\begin{array}{l}2.0 \pm 0.3 \mathrm{a} \\
39.3 \pm 6.3 \mathrm{~b} \\
- \\
1.2 \pm 0.2 \mathrm{a}, \mathrm{b} \\
- \\
- \\
32.4 \pm 4.6 \mathrm{a} \\
- \\
- \\
- \\
- \\
-\end{array}$ & $\begin{array}{l}0.031^{2} \\
0.003^{1} \\
0.002^{1} \\
0.006^{1} \\
0.001^{2} \\
- \\
0.001^{1} \\
0.065^{2} \\
0.043^{1} \\
- \\
<0.001^{2} \\
-\end{array}$ \\
\hline Sesquiterpenes & $\begin{array}{l}\alpha \text {-Cubebene } \\
\alpha \text {-Copaene } \\
\beta \text {-Bourbonene } \\
\beta \text {-Caryophyllene } \\
\alpha \text {-Caryophyllene } \\
\text { Germacrene D } \\
\alpha \text {-Farnesene } \\
\text { Valencene } \\
\Delta \text {-Cadinene }\end{array}$ & $\begin{array}{l}- \\
0.1 \pm 0.0 \mathrm{a} \\
- \\
0.6 \pm 0.3 \mathrm{a} \\
- \\
- \\
- \\
- \\
-\end{array}$ & $\begin{array}{l}\text { tr. } \\
0.3 \pm 0.1 \mathrm{a} \\
0.2 \pm 0.0 \mathrm{~b} \\
0.5 \pm 0.1 \mathrm{a} \\
0.2 \pm 0.0 \\
0.1 \pm 0.0 \\
- \\
- \\
0.1 \pm 0.0\end{array}$ & $\begin{array}{l}- \\
0.1 \pm 0.0 \mathrm{a} \\
0.1 \pm 0.0 \mathrm{a} \\
\text { tr. } \\
- \\
- \\
- \\
- \\
-\end{array}$ & $\begin{array}{l}- \\
\text { tr. } \\
- \\
0.2 \pm 0.1 \mathrm{a} \\
- \\
- \\
0.3 \pm 0.2 \\
\operatorname{tr} . \\
-\end{array}$ & $\begin{array}{l}- \\
\operatorname{tr} . \\
- \\
0.1 \pm 0.0 \mathrm{a} \\
- \\
- \\
- \\
- \\
-\end{array}$ & $\begin{array}{l}- \\
0.8 \pm 0.1 \mathrm{~b} \\
- \\
- \\
- \\
- \\
- \\
- \\
-\end{array}$ & $\begin{array}{l}- \\
<0.001^{2} \\
0.025^{2} \\
0.160^{2} \\
- \\
- \\
- \\
- \\
-\end{array}$ \\
\hline Terpenes & $\begin{array}{l}\alpha \text {-Pinene } \\
\rho \text {-Cymene } \\
\text { Limonene } \\
\beta \text {-Ocymene }\end{array}$ & $\begin{array}{l}- \\
- \\
0.8 \pm 0.2 \mathrm{a}, \mathrm{b} \\
-\end{array}$ & $\begin{array}{l}- \\
0.5 \pm 0.1 \mathrm{~b} \\
2.9 \pm 1.0 \mathrm{~b} \\
0.5 \pm 0.1\end{array}$ & $\begin{array}{l}0.1 \pm 0.0 \\
- \\
0.6 \pm 0.2 \mathrm{a} \\
-\end{array}$ & $\begin{array}{l}- \\
0.1 \pm 0.0 \mathrm{a} \\
0.1 \pm 0.0 \mathrm{a} \\
\text { tr. }\end{array}$ & $\begin{array}{l}- \\
- \\
0.2 \pm 0.0 \mathrm{a} \\
\text { tr. }\end{array}$ & $\begin{array}{l}- \\
- \\
1.8 \pm 0.73 a, b \\
-\end{array}$ & $\begin{array}{l}- \\
0.047^{2} \\
0.004^{1} \\
-\end{array}$ \\
\hline Aromatic hydrocarbons & $\begin{array}{l}\text { Toluene } \\
\rho \text {-Xylene } \\
\sigma \text {-Xylene }\end{array}$ & $\begin{array}{l}1.8 \pm 0.7 \mathrm{a} \\
2.0 \pm 0.7 \mathrm{a}, \mathrm{b} \\
-\end{array}$ & $\begin{array}{l}3.1 \pm 1.4 \mathrm{a}, \mathrm{b} \\
2.4 \pm 0.6 \mathrm{~b} \\
1.5 \pm 0.4 \mathrm{~b}\end{array}$ & $\begin{array}{l}8.0 \pm 1.8 \mathrm{~b}, \mathrm{c} \\
0.9 \pm 0.2 \mathrm{a}, \mathrm{b} \\
0.5 \pm 0.1 \mathrm{a}, \mathrm{b}\end{array}$ & $\begin{array}{l}3.5 \pm 0.1 \mathrm{a}, \mathrm{b} \\
0.4 \pm 0.0 \mathrm{a} \\
0.2 \pm 0.0 \mathrm{a}\end{array}$ & $\begin{array}{l}6.4 \pm 0.8 \mathrm{a}, \mathrm{b} \\
0.2 \pm 0.0 \mathrm{a} \\
\mathrm{tr}\end{array}$ & $\begin{array}{l}12.8 \pm 1.0 \mathrm{c} \\
- \\
-\end{array}$ & $\begin{array}{l}<0.001^{1} \\
0.007^{1} \\
0.015^{1}\end{array}$ \\
\hline
\end{tabular}

In the same line, mean values with different letters differ significantly $(P<0.05)$.

${ }^{1} P<0.05$, by means of Levene test. $P$ values are those from one-way Welch ANOVA analysis. Means were compared by Dunnett T3's test, since equal variances could not be assumed.

${ }^{2} P>0.05$, be means of Levene test. $P$ values are those from one-way ANOVA analysis. Means were compared by Tukey's test, since equal variances could be assumed.

Finally, three aromatic hydrocarbons were identified throughout the study: toluene, $\sigma$ - and $\rho$-xylene. Compared to toluene, $\sigma$ - and $\rho$-xylene were present in lower amounts. $\rho$-Xylene in cvs. Madural and Verdeal Transmontana increased from 18th Jul until 20th Sep, then decreased gradually until 21st Oct and was absent on 9th Nov (Tables 2 and 3). In cv. Cobrançosa, this aromatic hydrocarbon increased from 18th Jul to 18th Aug and afterward followed the same trend observed in the other two cultivars, a gradual decrease until 21st Oct and absence as of 9th Nov (Table 1). Toluene content represented a low percentage as of the first date (18th Jul) with $1.8 \%, 0.8 \%$, and $2.0 \%$ for cvs. Cobrançosa, Madural and Verdeal Transmontana, respectively (Tables 1-3). Meanwhile, maximum significant values were reported between 20th Sep and 4th Oct for cv. Madural (27.4\%; $P=0.014)$ and cv. Verdeal Transmontana (34.0\%; $P<0.001$ ), respectively. For cv. Cobrançosa, maximum values were reported later, on 9th Nov, reaching only $12.8 \%(P<0.001)$, a significantly lower value compared to the other two cultivars $(P<0.001)$. The volatile composition found in our cultivars is quite different from the composition reported by Scarpati et al. (1993). These authors reported the chromatographic profile of main volatiles found in olive leaves, but no information about the cultivar studied is provided in their work.

Considering the periods during which higher infestation levels were reported (4th and 21st Oct, and 9th Nov) and the most abundant volatiles present in those surveyed periods as well as total volatile emission $(\mu \mathrm{g} / 100 \mathrm{~g}$ olive leaf), PCA was applied
(Fig. 4). Fig. 4 shows that samples from cvs. Cobrançosa and Verdeal Transmontana are easily grouped, while samples from cv. Madural are mainly dispersed in the entire region of principal component 1 (PC1). Olive leaves from cv. Cobrançosa are mainly grouped in the positive regions of both principal components. Samples from 4th $\left(\mathrm{C}_{4}\right)$ and 21 st Oct $\left(\mathrm{C}_{5}\right)$ (fourth and fifth sampling dates) were mainly characterized by higher GLV content, including ( $Z$ )-3-hexen-1-ol and ( $Z$ )-3-hexen-1-ol acetate, and therefore, also, higher total volatile emissions as of these sampling dates. Cv. Verdeal Transmontana olive leaf samples, which emitted lower amounts of volatiles, appear in an extreme position. Samples of cv. Verdeal Transmontana from 9 th Nov. were characterized by high ester amounts, such as butanoic acid, 3-methyl-, methyl ester, and hexanoic acid methyl ester, as well as the monoterpene limonene. An interesting observation is that all the samples from $\mathrm{cv}$. Verdeal Transmontana are shown around the variable corresponding to infestation level, suggesting that this cultivar is clearly susceptible to olive fly oviposition. Toluene behavior is also shown at the extreme opposite of the samples from cv. Cobrançosa, and therefore close to the cv. Verdeal Transmontana olive leaf samples (Fig. 4).

\section{Discussion}

Secondary plant metabolites, such as volatiles, are metabolized through enzymatic pathways. Their formation is therefore 
Table 2

Volatile composition (\%; mean \pm standard error) of $\mathrm{cv}$. Madural olive leaf at different harvest times.

\begin{tabular}{|c|c|c|c|c|c|c|c|c|}
\hline Chemical class & Compound & 18th Jul & 18th Aug & 20th Sep & 4th Oct & 21st Oct & 9th Nov & $P$-value \\
\hline Alcohols & $\begin{array}{l}\text { 3-Methyl-1-butanol } \\
\text { 3-Hexanol } \\
\text { (Z)-3-Hexen-1-ol }\end{array}$ & $\begin{array}{l}0.4 \pm 0.1 \mathrm{a} \\
- \\
1.3 \pm 0.4 \mathrm{a}\end{array}$ & $\begin{array}{l}0.4 \pm 0.1 \mathrm{a} \\
2.6 \pm 0.9 \mathrm{~b} \\
1.9 \pm 0.7 \mathrm{a}\end{array}$ & $\begin{array}{l}0.3 \pm 0.0 \mathrm{a} \\
1.0 \pm 0.3 \mathrm{a}, \mathrm{b} \\
2.2 \pm 0.5 \mathrm{a}\end{array}$ & $\begin{array}{l}- \\
0.3 \pm 0.2 \mathrm{a} \\
4.7 \pm 1.4 \mathrm{a}\end{array}$ & $\begin{array}{l}- \\
- \\
12.5 \pm 6.6 \mathrm{a}\end{array}$ & $\begin{array}{l}- \\
- \\
6.1 \pm 1.5 \mathrm{a}\end{array}$ & $\begin{array}{l}0.605^{2} \\
0.050^{2} \\
0.116^{1}\end{array}$ \\
\hline Aldehydes & $\begin{array}{l}\text { Hexanal } \\
\text { Nonanal } \\
\text { Decanal }\end{array}$ & $\begin{array}{l}- \\
- \\
-\end{array}$ & $\begin{array}{l}- \\
0.6 \pm 0.2 \mathrm{a} \\
0.3 \pm 0.0 \mathrm{a}\end{array}$ & $\begin{array}{l}0.5 \pm 0.1 \\
0.3 \pm 0.0 \mathrm{a} \\
-\end{array}$ & $\begin{array}{l}- \\
0.2 \pm 0.1 \mathrm{a} \\
0.2 \pm 0.1 \mathrm{a}\end{array}$ & $\begin{array}{l}- \\
- \\
-\end{array}$ & $\begin{array}{l}- \\
- \\
-\end{array}$ & $\begin{array}{l}- \\
0.136^{1} \\
0.128^{2}\end{array}$ \\
\hline Esters & $\begin{array}{l}\text { Butanoic acid methyl ester } \\
\text { Butanoic acid, 3-methyl-, methyl ester } \\
\text { Butanoic acid, 2-methyl-, methyl ester } \\
\text { Hexanoic acid methyl ester } \\
\text { (Z)-3-Hexenoic acid methyl ester } \\
\text { (Z)-3-Hexen-1-ol acetate } \\
\text { Hexyl acetate } \\
\text { Benzoic acid methyl ester } \\
\text { (Z)-3-Hexenyl isobutyrate } \\
\text { (Z)-3-Hexenyl butyrate }\end{array}$ & $\begin{array}{l}5.9 \pm 1.4 \mathrm{a} \\
6.5 \pm 1.7 \mathrm{a} \\
26.5 \pm 2.9 \mathrm{c} \\
- \\
- \\
49.7 \pm 6.6 \mathrm{a} \\
4.1 \pm 1.0 \mathrm{~b} \\
3.0 \pm 1.6 \mathrm{a} \\
- \\
-\end{array}$ & $\begin{array}{l}5.7 \pm 0.5 \mathrm{a} \\
1.3 \pm 0.5 \mathrm{a} \\
22.2 \pm 6.9 \mathrm{~b}, \mathrm{c} \\
0.5 \pm 0.1 \mathrm{a} \\
- \\
45.9 \pm 10.5 \mathrm{a} \\
1.3 \pm 0.4 \mathrm{a} \\
4.4 \pm 1.3 \mathrm{a} \\
- \\
-\end{array}$ & $\begin{array}{l}4.8 \pm 1.4 \mathrm{a} \\
1.5 \pm 0.5 \mathrm{a} \\
15.5 \pm 5.0 \mathrm{a}-\mathrm{c} \\
- \\
- \\
32.8 \pm 6.8 \mathrm{a} \\
2.3 \pm 0.5 \mathrm{a}, \mathrm{b} \\
3.2 \pm 1.2 \mathrm{a} \\
- \\
-\end{array}$ & $\begin{array}{l}21.2 \pm 10.6 \mathrm{~b} \\
- \\
4.0 \pm 0.8 \mathrm{a} \\
0.5 \pm 0.1 \mathrm{a} \\
0.7 \pm 0.3 \\
40.2 \pm 11.8 \mathrm{a} \\
0.7 \pm 0.3 \mathrm{a} \\
4.5 \pm 1.3 \mathrm{a} \\
0.4 \pm 0.2 \\
3.2 \pm 1.3\end{array}$ & $\begin{array}{l}4.1 \pm 1.0 \mathrm{a} \\
1.2 \pm 0.6 \mathrm{a} \\
5.2 \pm 1.2 \mathrm{a}, \mathrm{b} \\
0.5 \pm 0.1 \mathrm{a} \\
- \\
52.3 \pm 5.7 \mathrm{a} \\
1.2 \pm 0.3 \mathrm{a} \\
1.3 \pm 0.4 \mathrm{a} \\
- \\
-\end{array}$ & $\begin{array}{l}7.2 \pm 0.3 \mathrm{a} \\
9.0 \pm 5.1 \mathrm{a} \\
11.8 \pm 2.4 \mathrm{a}-\mathrm{c} \\
1.4 \pm 0.3 \mathrm{~b} \\
- \\
38.6 \pm 12.3 \mathrm{a} \\
- \\
- \\
- \\
-\end{array}$ & $\begin{array}{l}0.030^{2} \\
0.166^{1} \\
0.002^{1} \\
0.013^{2} \\
- \\
0.685^{2} \\
0.005^{2} \\
0.378^{2} \\
- \\
-\end{array}$ \\
\hline Ketones & 6-Methyl-5-hepten-2-one & - & $0.7 \pm 0.2$ & - & - & - & - & - \\
\hline Sesquiterpenes & $\begin{array}{l}\alpha \text {-Copaene } \\
\beta \text {-Caryophyllene } \\
\alpha \text {-Caryophyllene } \\
\alpha \text {-Farnesene }\end{array}$ & $\begin{array}{l}0.2 \pm 0.0 \mathrm{a} \\
0.4 \pm 0.1 \mathrm{a} \\
- \\
-\end{array}$ & $\begin{array}{l}0.1 \pm 0.0 \mathrm{a} \\
0.2 \pm 0.0 \mathrm{a} \\
0.1 \pm 0.0 \\
0.5 \pm 0.2\end{array}$ & $\begin{array}{l}- \\
0.2 \pm 0.0 \mathrm{a} \\
- \\
-\end{array}$ & $\begin{array}{l}0.1 \pm 0.0 \mathrm{a} \\
0.2 \pm 0.1 \mathrm{a} \\
- \\
-\end{array}$ & $\begin{array}{l}0.1 \pm 0.1 \mathrm{a} \\
0.1 \pm 0.0 \mathrm{a} \\
- \\
-\end{array}$ & $\begin{array}{l}- \\
0.3 \pm 0.1 \text { a } \\
- \\
-\end{array}$ & $\begin{array}{l}0.855^{2} \\
0.242^{2} \\
- \\
-\end{array}$ \\
\hline Terpenes & $\begin{array}{l}\alpha \text {-Pinene } \\
\rho \text {-Cymene } \\
\text { Limonene } \\
\beta \text {-Ocymene }\end{array}$ & $\begin{array}{l}- \\
- \\
0.4 \pm 0.1 \mathrm{a} \\
-\end{array}$ & $\begin{array}{l}- \\
0.5 \pm 0.1 \mathrm{a} \\
3.0 \pm 1.0 \mathrm{~b} \\
1.8 \pm 1.1 \mathrm{a}\end{array}$ & $\begin{array}{l}0.2 \pm 0.0 \\
- \\
0.8 \pm 0.2 \mathrm{a} \\
-\end{array}$ & $\begin{array}{l}- \\
0.5 \pm 0.2 \mathrm{a} \\
0.4 \pm 0.2 \mathrm{a} \\
0.4 \pm 0.2 \mathrm{a}\end{array}$ & $\begin{array}{l}- \\
- \\
0.3 \pm 0.1 \mathrm{a} \\
-\end{array}$ & $\begin{array}{l}- \\
- \\
1.9 \pm 0.5 \mathrm{a}, \mathrm{b} \\
-\end{array}$ & $\begin{array}{l}- \\
0.912^{2} \\
0.004^{2} \\
0.265^{2}\end{array}$ \\
\hline Aromatic hydrocarbons & $\begin{array}{l}\text { Toluene } \\
\rho \text {-Xylene } \\
\sigma \text {-Xylene }\end{array}$ & $\begin{array}{l}0.8 \pm 0.2 \mathrm{a} \\
0.9 \pm 0.2 \mathrm{a} \\
-\end{array}$ & $\begin{array}{l}2.1 \pm 0.7 \mathrm{a} \\
2.2 \pm 0.6 \mathrm{a}, \mathrm{b} \\
1.5 \pm 0.4 \mathrm{a}\end{array}$ & $\begin{array}{l}27.4 \pm 4.5 \mathrm{c} \\
4.3 \pm 1.0 \mathrm{~b} \\
2.4 \pm 0.5 \mathrm{a}\end{array}$ & $\begin{array}{l}14.2 \pm 7.3 b \\
2.0 \pm 0.9 a, b \\
1.4 \pm 0.7 a\end{array}$ & $\begin{array}{l}20.7 \pm 7.4 b \\
0.4 \pm 0.1 \mathrm{a} \\
-\end{array}$ & $\begin{array}{l}23.7 \pm 7.9 \mathrm{~b} \\
- \\
-\end{array}$ & $\begin{array}{l}0.014^{2} \\
0.007^{2} \\
0.372^{2}\end{array}$ \\
\hline
\end{tabular}

In the same line, mean values with different letters differ significantly $(P<0.05)$.

${ }^{1} P<0.05$, by means of Levene test. $P$ values are those from one-way Welch ANOVA analysis. Means were compared by Dunnett T3's test, since equal variances could not be assumed.

${ }^{2} P>0.05$, be means of Levene test. $P$ values are those from one-way ANOVA analysis. Means were compared by Tukey's test, since equal variances could be assumed.

monitored by enzymes and influenced by several important factors, including the cultivar (Brahmi et al., 2012), time of year (Campeol et al., 2003), and region. Therefore, the quantitative and qualitative changes observed in this work are mainly related to cultivar specificities and the harvest time assessed, as the olive trees surveyed were all cultivated in the same olive grove. Our results highlight the presence of qualitative and quantitative differences among the volatile compositions of the different cultivars assayed as well as the harvest times assessed.

The main compounds found in the volatile fractions (GLVs) of the three cultivars are formed through the lipoxygenase pathway (LOX). The hydrolysis of polyunsaturated fatty acids (linoleic and $\alpha$-linolenic acids) present in olive leaves is conducted by endogenous acyl-hydrolases, leading to the formation of free acids, modified by lipoxygenase enzymes at chain positions C9 and C13 and forming 9- and 13-hydroperoxydes. These fatty acid derivatives are then metabolized and excised by hydroperoxide lyases, leading to the formation of short-chain C6 aldehydes, such as (Z)-3hexenal and hexanal. These C6 aldehydes are unstable and, spontaneously or by enzymatic action (enal-isomerases), can be converted to positional isomers or reduced to alcohols by alcohol dehydrogenases. These C6 alcohols can be then esterified by alcohol acyltransferases to yield volatile esters, e.g., ( $Z$ )-3-hexen1-ol is esterified to (Z)-3-hexen-1-ol acetate (Akacha and Gargouri, 2009; Dudareva et al., 2006; Salas et al., 2005). The high contents of $(Z)$-3-hexen-1-ol acetate and (Z)-3-hexen-1-ol found in olive leaf volatile fractions, rather than hexanol and hexyl acetate, are plausible because olive leaves are three times richer in linolenic acid than in linoleic acid (Guerfel et al., 2008), with higher activity in the LOX linoleic acid branch reported.

During the surveyed period, the reduction of C6 esters and GLVs (more specifically $(Z$ )-3-hexen-1-ol acetate) may be related to the
LOX pathway. We hypothesize that lipoxygenase activity in olive leaves can be significantly influenced by the climatic conditions. Lipoxygenase is more active in stressed plants and drought conditions (Sofo et al., 2004). Therefore, high levels of GLVs as of the first dates could be due to higher lipoxygenase activity, as the plants were in drought conditions for a long period. During collection, mainly during the end of September and beginning of October, with increasing rainfall, GLVs and (Z)-3-hexen-1-ol acetate contents decrease drastically, because lipoxygenase can reduce its activity threefold in plants experiencing water availability (Sofo et al., 2004). Furthermore, LOX substrate ( $\alpha$-linoleic free fatty acids) could also be reduced, as olive leaves from irrigated olive trees reduce their linoleic acid content (Guerfel et al., 2008), thus influencing the LOX mechanisms. In olives from the same cultivars, GLV content is also reduced during fruit maturation, in relation to the ripening process (Malheiro et al., 2015b).

A contrasting trend to GLV's was observed for the aromatic hydrocarbon toluene. Toluene has been previously described in olive oil and table olives (Baccouri et al., 2008; Iraqui et al., 2005). The origin of toluene in olive leaves and olive food products remains unknown. Some authors claim that the presence of toluene in plants can be derived from exogenous contamination, as well as from endogenous mechanisms (Biedermann et al., 1995), while others claim that toluene emission is enhanced in stressed plants (Heiden et al., 1999). However, in our study, the increase in toluene was observed during the period where olive tree was less stressed due to rainfall. In fact, in this study, rainfall may be related to the significant increase in toluene in the olive leaf volatile composition. Some authors have shown that aromatic hydrocarbon formation is considerably enhanced by epiphytic microorganisms present in the olive leaf surface during late summer rainfall (Scarpati et al., 1996). These authors verified the 
Table 3

Volatile composition (\%; mean \pm standard error) of cv. Verdeal Transmontana olive leaf at different harvest times.

\begin{tabular}{|c|c|c|c|c|c|c|c|c|}
\hline Chemical class & Compound & 18th Jul & 18th Aug & 20th Sep & 4th Oct & 21 st Oct & 9th Nov & $P$-value \\
\hline Alcohols & $\begin{array}{l}\text { 3-Methyl-1-butanol } \\
\text { 3-Hexanol } \\
\text { (Z)-3-Hexen-1-ol } \\
\text { Hexanol } \\
\text { Octanol }\end{array}$ & $\begin{array}{l}- \\
- \\
5.6 \pm 0.7 \mathrm{a} \\
- \\
-\end{array}$ & $\begin{array}{l}15.1 \pm 8.1 b \\
2.0 \pm 1.5 \mathrm{a} \\
19.0 \pm 14.2 \mathrm{~b} \\
1.2 \pm 0.8 \\
0.1 \pm 0.0\end{array}$ & $\begin{array}{l}0.9 \pm 0.2 \mathrm{a} \\
0.8 \pm 0.3 \mathrm{a} \\
4.3 \pm 1.4 \mathrm{a} \\
- \\
-\end{array}$ & $\begin{array}{l}- \\
- \\
7.0 \pm 0.7 \mathrm{a} \\
- \\
-\end{array}$ & $\begin{array}{l}- \\
- \\
20.6 \pm 8.7 \mathrm{~b} \\
- \\
-\end{array}$ & $\begin{array}{l}- \\
- \\
12.6 \pm 4.2 \mathrm{a}, \mathrm{b} \\
- \\
-\end{array}$ & $\begin{array}{l}0.030^{2} \\
0.498^{1} \\
<0.001^{2} \\
- \\
-\end{array}$ \\
\hline Aldehydes & $\begin{array}{l}\text { Nonanal } \\
\text { Decanal }\end{array}$ & - & $\begin{array}{l}0.5 \pm 0.1 \mathrm{a} \\
0.3 \pm 0.1\end{array}$ & $\begin{array}{l}0.2 \pm 0.0 \mathrm{a} \\
-\end{array}$ & - & - & - & $\begin{array}{l}0.095^{2} \\
-\end{array}$ \\
\hline Esters & $\begin{array}{l}\text { Butanoic acid methyl ester } \\
\text { Butanoic acid, 3-methyl-, methyl ester } \\
\text { Butanoic acid, 2-methyl-, methyl ester } \\
\text { Hexanoic acid methyl ester } \\
\text { (Z)-3-Hexenoic acid methyl ester } \\
\text { (Z)-3-Hexen-1-ol acetate } \\
\text { Hexyl acetate } \\
\text { Benzoic acid methyl ester } \\
\text { (Z)-3-Hexenyl butyrate }\end{array}$ & $\begin{array}{l}7.5 \pm 2.0 \mathrm{a} \\
5.4 \pm 1.6 \mathrm{~b} \\
13.6 \pm 2.3 \mathrm{~b} \\
- \\
- \\
50.4 \pm 8.3 \mathrm{~b} \\
1.3 \pm 0.5 \mathrm{a} \\
5.9 \pm 2.1 \mathrm{~b} \\
-\end{array}$ & $\begin{array}{l}2.8 \pm 1.1 \mathrm{a} \\
0.9 \pm 0.4 \mathrm{a} \\
2.5 \pm 0.9 \mathrm{a} \\
0.5 \pm 0.1 \mathrm{a} \\
- \\
40.2 \pm 16.9 \mathrm{a}, \mathrm{b} \\
0.9 \pm 0.4 \mathrm{a} \\
1.4 \pm 0.8 \mathrm{a} \\
-\end{array}$ & $\begin{array}{l}7.9 \pm 4.8 \mathrm{a} \\
4.8 \pm 2.2 \mathrm{~b} \\
7.9 \pm 1.6 \mathrm{a}, \mathrm{b} \\
1.0 \pm 0.2 \mathrm{a} \\
- \\
27.4 \pm 1.7 \mathrm{a}, \mathrm{b} \\
- \\
2.2 \pm 0.2 \mathrm{a} \\
-\end{array}$ & $\begin{array}{l}8.0 \pm 0.1 \mathrm{a} \\
1.1 \pm 0.0 \mathrm{a} \\
7.2 \pm 1.9 \mathrm{a}, \mathrm{b} \\
1.2 \pm 0.2 \mathrm{a}, \mathrm{b} \\
0.6 \pm 0.1 \\
21.1 \pm 4.2 \mathrm{a}, \mathrm{b} \\
- \\
13.6 \pm 0.4 \mathrm{c} \\
1.8 \pm 0.1\end{array}$ & $\begin{array}{l}8.9 \pm 1.3 \mathrm{a} \\
1.7 \pm 0.4 \mathrm{a} \\
6.3 \pm 2.8 \mathrm{a}, \mathrm{b} \\
- \\
- \\
22.9 \pm 4.2 \mathrm{a}, \mathrm{b} \\
- \\
9.4 \pm 3.5 \mathrm{~b}, \mathrm{c} \\
-\end{array}$ & $\begin{array}{l}5.1 \pm 2.0 \mathrm{a} \\
33.6 \pm 5.5 \mathrm{c} \\
3.4 \pm 0.9 \mathrm{a} \\
2.1 \pm 0.4 \mathrm{~b} \\
- \\
10.4 \pm 3.0 \mathrm{a} \\
- \\
- \\
-\end{array}$ & $\begin{array}{l}0.051^{1} \\
0.007^{1} \\
0.005^{2} \\
0.004^{2} \\
- \\
0.013^{1} \\
0.889^{2} \\
<0.001^{2} \\
-\end{array}$ \\
\hline Ketones & $\begin{array}{l}\text { 3-Pentanone } \\
\text { 3-Hexanone } \\
\text { 6-Methyl-5-hepten-2-one }\end{array}$ & $\begin{array}{l}3.9 \pm 1.1 \mathrm{a} \\
- \\
-\end{array}$ & $\begin{array}{l}4.2 \pm 2.1 \mathrm{a} \\
1.1 \pm 0.8 \\
1.2 \pm 0.6\end{array}$ & $\begin{array}{l}- \\
- \\
-\end{array}$ & $\begin{array}{l}- \\
- \\
-\end{array}$ & $\begin{array}{l}- \\
- \\
-\end{array}$ & $\begin{array}{l}- \\
- \\
-\end{array}$ & $\begin{array}{l}0.890^{2} \\
- \\
-\end{array}$ \\
\hline Sesquiterpenes & $\begin{array}{l}\alpha \text {-Copaene } \\
\beta \text {-Bourbonene } \\
\beta \text {-Caryophyllene } \\
\text { Valencene } \\
\Delta \text {-Cadinene }\end{array}$ & $\begin{array}{l}0.4 \pm 0.2 \mathrm{a} \\
- \\
1.1 \pm 0.4 \mathrm{~b} \\
- \\
-\end{array}$ & $\begin{array}{l}- \\
0.2 \pm 0.1 \\
0.1 \pm 0.0 \mathrm{a} \\
- \\
-\end{array}$ & $\begin{array}{l}- \\
- \\
0.2 \pm 0.1 \mathrm{a} \\
0.1 \pm 0.0 \mathrm{a} \\
0.2 \pm 0.0\end{array}$ & $\begin{array}{l}- \\
- \\
0.3 \pm 0.0 \mathrm{a} \\
0.6 \pm 0.2 \mathrm{~b} \\
-\end{array}$ & $\begin{array}{l}0.2 \pm 0.1 \mathrm{a} \\
- \\
0.3 \pm 0.1 \mathrm{a} \\
- \\
-\end{array}$ & $\begin{array}{l}- \\
- \\
0.4 \pm 0.1 \mathrm{a} \\
- \\
-\end{array}$ & $\begin{array}{l}0.273^{2} \\
- \\
0.003^{1} \\
0.016^{2} \\
-\end{array}$ \\
\hline Terpenes & $\begin{array}{l}\alpha \text {-Pinene } \\
\text { Limonene } \\
\beta \text {-Ocymene }\end{array}$ & $\begin{array}{l}- \\
1.4 \pm 0.5 \mathrm{a} \\
-\end{array}$ & $\begin{array}{l}- \\
1.5 \pm 0.6 \mathrm{a} \\
0.3 \pm 0.1 \mathrm{a}\end{array}$ & $\begin{array}{l}0.3 \pm 0.1 \\
2.1 \pm 0.6 \mathrm{a} \\
-\end{array}$ & $\begin{array}{l}- \\
0.7 \pm 0.1 \mathrm{a} \\
0.3 \pm 0.1 \mathrm{a}\end{array}$ & $\begin{array}{l}- \\
0.3 \pm 0.1 \mathrm{a} \\
-\end{array}$ & $\begin{array}{l}- \\
8.1 \pm 1.8 \mathrm{~b} \\
-\end{array}$ & $\begin{array}{l}- \\
<0.001^{2} \\
0.833^{2}\end{array}$ \\
\hline Aromatic hydrocarbons & $\begin{array}{l}\text { Toluene } \\
\rho \text {-Xylene } \\
\sigma \text {-Xylene }\end{array}$ & $\begin{array}{l}2.0 \pm 1.1 \mathrm{a} \\
1.7 \pm 0.6 \mathrm{a}, \mathrm{b} \\
-\end{array}$ & $\begin{array}{l}1.5 \pm 0.5 \mathrm{a} \\
1.3 \pm 0.5 \mathrm{a} \\
0.9 \pm 0.4 \mathrm{a}\end{array}$ & $\begin{array}{l}34.2 \pm 6.0 \mathrm{c} \\
3.6 \pm 0.7 \mathrm{~b} \\
1.9 \pm 0.3 \mathrm{a}\end{array}$ & $\begin{array}{l}34.0 \pm 3.6 \mathrm{c} \\
1.2 \pm 0.2 \mathrm{a} \\
1.2 \pm 0.2 \mathrm{a}\end{array}$ & $\begin{array}{l}27.9 \pm 7.1 \mathrm{~b}, \mathrm{c} \\
0.2 \pm 0.1 \mathrm{a} \\
-\end{array}$ & $\begin{array}{l}24.2 \pm 6.8 b \\
- \\
-\end{array}$ & $\begin{array}{l}<0.001^{1} \\
0.003^{2} \\
0.143^{2}\end{array}$ \\
\hline
\end{tabular}

In the same line, mean values with different letters differ significantly $(P<0.05)$.

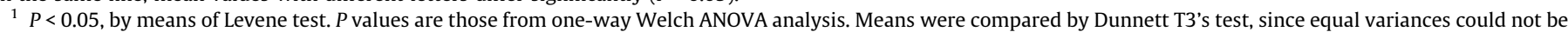
assumed.

${ }^{2} P>0.05$, be means of Levene test. $P$ values are those from one-way ANOVA analysis. Means were compared by Tukey's test, since equal variances could be assumed.

attraction of the olive fly to olive orchards after the first summer rainfall. The same research group showed that the aromatic hydrocarbons styrene and toluene are oviposition attractants to the olive fly (Scarpati et al., 1993, 1996). In our work, we found a positive correlation between toluene abundance in the olive leaf from the three olive cultivars and the infestation levels of the respective olive fruits $\left(R^{2}=0.249 ; P<0.001 ; y=0.31 x+8.04\right)$. Therefore, higher toluene contents could be associated with higher infestation levels, possibly related to the oviposition attractant properties of this aromatic hydrocarbon, as demonstrated by Scarpati et al. (1993) and Scarpati et al. (1996). The same fact was verified in the olives of the three olive cultivars studied (Malheiro et al., 2015b). Toluene released from olives was correlated with the infestation levels verified in cvs. Verdeal Transmontana and Madural, while no correlations were obtained in cv. Cobrançosa. Therefore toluene could intervene in the oviposition preference of olive fly females.

Another compound with important attractant activity to the olive fly is the monoterpene limonene. This compound was present in higher amounts in cv. Verdeal Transmontana than in cvs. Cobrançosa and Madural, especially on 9th Nov. The R-enantiomer of limonene, R-(+)-limonene, is highly attractive to both olive fly sexes in wind tunnel assays (De Cristofaro et al., 2007).

The host selection of tephritid fruit flies is influenced by several factors, among which volatiles display an important role (Aluja and Mangan, 2008). Our hypothesis is that volatiles emitted by olive leaves may interfere in olive fly females' host selection in conjunction with olives (Malheiro et al., 2015b) and with other factors, such as physical (fruit and leaf color, shape, volume) (Rizzo et al., 2012) and biochemical aspects (olives maturation) (Gonçalves et al., 2012). In fact, when host volatiles from olive leaves and fruits were tested in electroantenographic studies in olive fly males and females (mated and unmated), higher EAG signals were obtained with olive leaf volatiles, especially in mated males (Liscia et al., 2013). The results obtained in Liscia et al. (2013) indicate that host volatiles are recognized by the olive fly, and their recognition is higher for olive leaf volatiles rather than olives. As volatiles are highly influenced by olive cultivar, olive leaf volatiles are likely to be important cues in host selection. A second factor observed was the volatile amounts, which were clearly higher in cv. Cobrançosa olive leaves, most likely representing a deterrent factor against other less intensive emissions from the other cultivars. Recent studies revealed that ethylene production is induced by olive fly larva when they feed in the olive pulp (Alagna et al., 2015). The production of ethylene by olives could give important information from an ecological point of view. Ethylene production could alert olive flies to the presence of larvae in the olives, leading the olive fly females to oviposit in other olives, increasing therefore the probability of larvae to complete their cycle and the creation of new generations of olive flies. By other hand, ethylene production may increase during olives maturation and could also indicate to olive flies that olive are ripe and the probability of larvae to complete their cycle is lower. This could be a co-evolution phenomenon observed in olive orchards.

\section{Conclusions}

In conclusion, the olive fly exhibits a cultivar preference towards cv. Verdeal Transmontana and Madural over cv. Cobrançosa. 

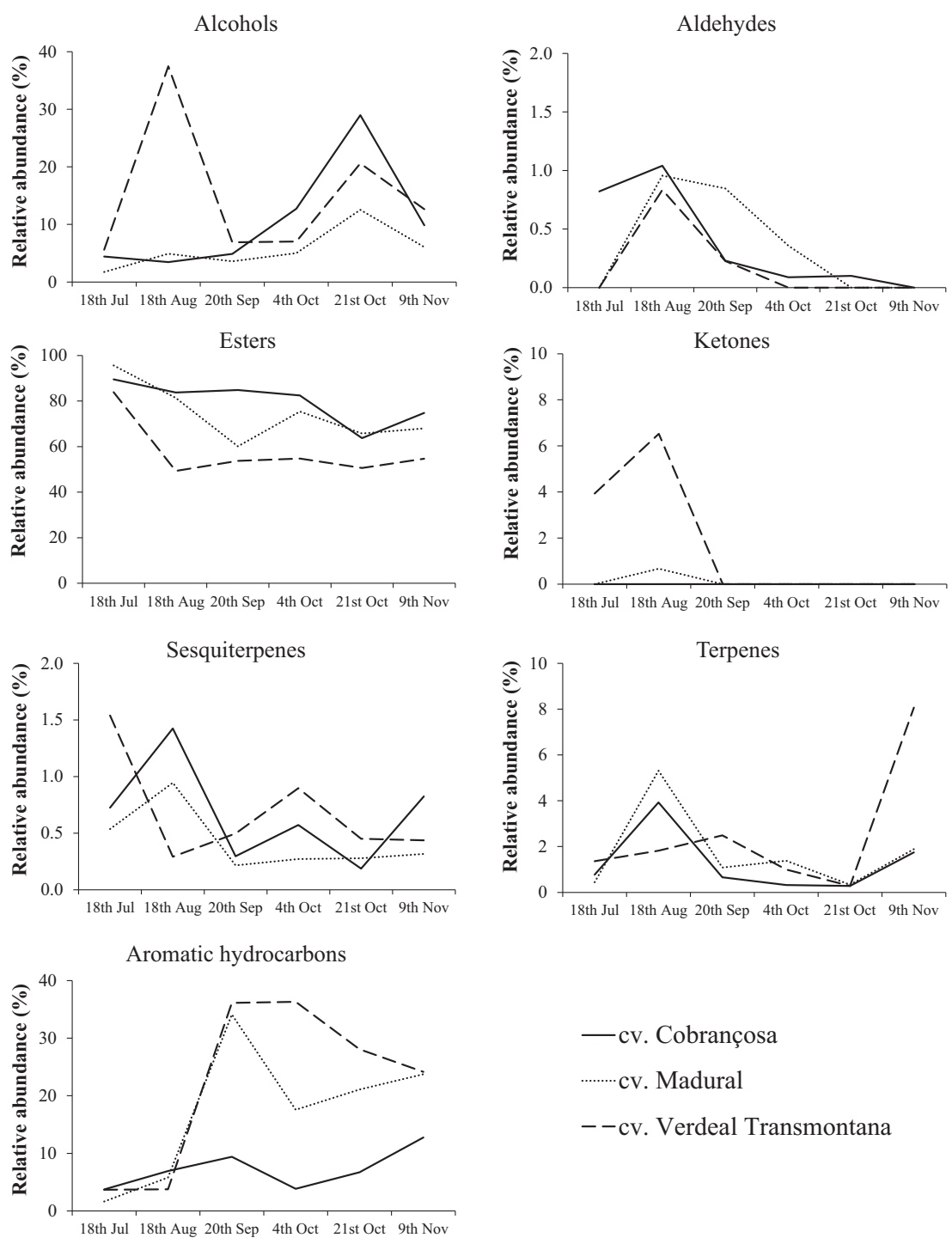

- cv. Cobrançosa

.......... cv. Madural

$--c v$. Verdeal Transmontana

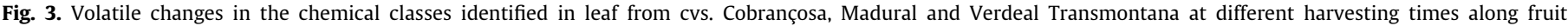
maturation (within the same cultivar, mean values with different letters during dates differ significantly, $P<0.05$ )

The volatile types and amounts emitted by olive leave are dependent on the olive cultivar and undergo considerable changes with the various harvest times surveyed. Olive leaf volatiles consist mainly of esters, alcohols and the aromatic hydrocarbon toluene. For the olive cultivars studied, correlations between toluene and infestation levels during olive maturation were reported. This compound may play an important role in attractiveness to the olive fly and in the cultivar preference of this olive pest. Olive leaf volatiles may act as a short-range cue for the olive fly, interacting with other factors (both chemical and physical cues) and influencing the host selection by the olive fly.

\section{Experimental}

\subsection{Sampling}

For this study, olive leaves from three Portuguese olive cultivars, the most representative from the Trás-os-Montes region (Northeast of Portugal), were assessed: cvs. Cobrançosa, Madural and Verdeal Transmontana. The work was performed in 2011, and samples were collected in an organic olive grove (not even chemicals allowed in organic farming were applied for the control of the olive fly) located in Paradela (Mirandela - 41 $32^{\prime} 35.72^{\prime \prime} \mathrm{N}$; $7^{\circ} 07^{\prime} 27.17^{\prime \prime} \mathrm{W}$ ). Five trees were marked per olive cultivar, and olive branches with leaves were collected at six different dates: 18th July; 18th August; 20th September; 4th October; 21st October; and 9th November. After collection, branches were transported at refrigeration temperatures, and volatile analysis was performed in the first 24-48 h.

Simultaneously, fruits were collected per tree for calculation of the maturation index, as described by Hermoso et al. (2001). Briefly, samples of 100 olive fruits (20 fruits per tree) were separated into 8 levels based on epidermis and pulp color (0 to 7). The fruit is classified as " 0 " if the epidermis is green; " 1 " for yellowish green; "2" if the epidermis shows red spots on less than half the fruit; " 3 " if the epidermis is red or purple on more than half the fruit; " 4 " for black epidermis and white pulp; " 5 " if the epidermis is black and less than half the pulp is purple; " 6 " if the epidermis is black and more than half the pulp purple (without reaching the stone); " 7 " if the epidermis is black and the whole pulp purple (reaching the stone). The maturation index was calculated as follows: $\quad \mathrm{MI}=(a \times 0+b \times 1+c \times 2+d \times 3+e \times 4+f \times 5+g \times 6+$ $h \times 7) / 100$, where the letters are the number of fruits in each level of classification considered. 


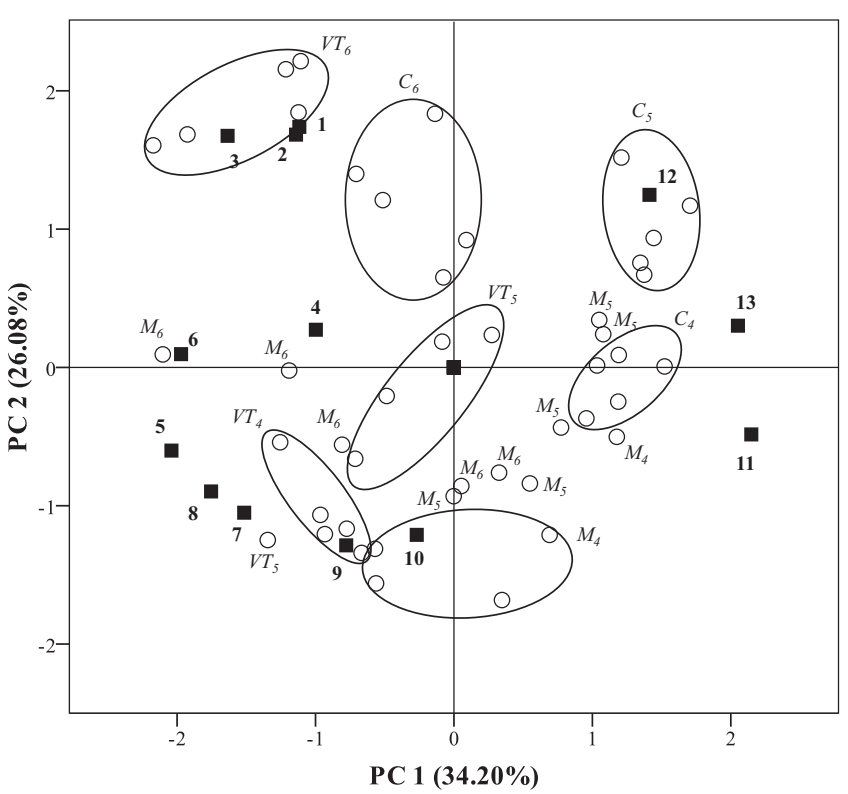

Fig. 4. Principal component analysis obtained from the main volatile compounds of olive leaf from cvs. Cobrançosa, Madural, and Verdeal Transmontana at different harvesting times along fruit maturation (4th Oct $\left(\mathrm{C}_{4}, \mathrm{M}_{4}\right.$ and $\left.\mathrm{VT}_{4}\right) ; 21$ st Oct $\left(\mathrm{C}_{5}, \mathrm{M}_{5}\right.$ and $\left.\mathrm{VT}_{5}\right)$; 9 th $\operatorname{Nov}\left(\mathrm{C}_{6}, \mathrm{M}_{6}\right.$ and $\left.\mathrm{VT}_{6}\right)$ ). 1 - Butanoic acid, 3-methyl- methyl ester; 2 hexanoic acid methyl ester; 3 - limonene; 4 - infestation level; 5 - toluene; 6 - $\beta$ caryophyllene; 7 - butanoic acid, 2-methyl-methyl ester; 8 - benzoic acid methyl ester; 9 - $\rho$-xylene; 10 - butanoic acid methyl ester; 11 - $(Z)$-3-hexen-1-ol acetate; 12 - (Z)-3-hexen-1-ol; 13 - total volatiles ( $\mu \mathrm{g}$ of volatiles/100 $\mathrm{g}$ of olive leaf). The principal components (PC) explain $60.28 \%$ of the total variance.

To assess infestation level, from 4th August to 23rd November 20 , random handpicked fruits from each olive tree 5 trees per cultivar; 100 fruits) were collected fortnightly and inspected in a binocular stereomicroscope for signs of infestation (oviposition sites or exit holes). Infestation level was expressed as the percentage of infested olive fruits.

\subsection{Volatile characterization}

\subsubsection{SPME fibers}

For headspace solid-phase microextraction (HS-SPME) a fiber coated with divinylbenzene/carbonex/polydimethylsiloxane (DVB/CAR/PDMS), 50/30 $\mu \mathrm{m}$ was selected based on a preliminary assay conducted alongside two other fibers (CAR/PDMS $75 \mu \mathrm{m}$ and PDMS $100 \mu \mathrm{m}$ ), all from Supelco (Bellefonte, USA). Selection of the fiber was based on the highest qualitative (number of volatiles extracted) and quantitative data (peak areas) of a sample of olive leaves from cv. Cobrançosa.

\subsubsection{HS-SPME}

HS-SPME was conducted according to the methodology applied by our research group in other matrices (Malheiro et al., 2013), with some modifications. Five healthy leaves (approximately $1 \mathrm{~g}$ ) were placed in $50 \mathrm{ml}$ vials. Deuterated chloroform (99.96\%, Aldrich) was added as internal standard (250 ppm in methanol; $10 \mu \mathrm{l}$ ), and the vial was immediately sealed with a polypropylene cap with a silicon septum. The volatiles were released at $40^{\circ} \mathrm{C}$ for $30 \mathrm{~min}$ in an ultrasonic bath. Next, the DVB/CAR/PDMS fiber was exposed for $30 \mathrm{~min}$ at $40^{\circ} \mathrm{C}$ for volatile adsorption, then inserted into the injection port of the GC system for thermal desorption and reconditioning $\left(10 \mathrm{~min}\right.$ at $280^{\circ} \mathrm{C}$ ). For each harvest time and olive cultivar, the HS-SPME analysis was performed in quintuplicate.

\subsubsection{Gas chromatography-mass spectrometry (GC-MS) conditions}

Chromatographic analysis was performed using an Agilent 6890 series GC (Agilent, Avondale, PA, USA) with splitless injection, coupled to a MS detector (Agilent 5973). Volatiles were separated using a bounded phase fused-silica capillary column (SPB-5, $60 \mathrm{~m} \times 0.32 \mathrm{~mm} \times 1 \mu \mathrm{m}$, Supelco, Bellefonte, USA) operating at constant flow with helium at $1 \mathrm{ml} \mathrm{min}^{-1}$. The oven temperature program was isothermal for $5 \mathrm{~min}$ at $40^{\circ} \mathrm{C}$, raised to $220^{\circ} \mathrm{C}$ at a rate of $3{ }^{\circ} \mathrm{C} \mathrm{min}-1$ and maintained at $220^{\circ} \mathrm{C}$ for $2 \mathrm{~min}$, with a total run time of $67 \mathrm{~min}$. The transfer line to the mass spectrometer was maintained at $250^{\circ} \mathrm{C}$. Mass spectra were obtained by electronic impact at $70 \mathrm{eV}$ with a multiplier voltage of $2056 \mathrm{~V}$, collecting data at a rate of $1 \mathrm{scan} \mathrm{s}^{-1}$ over the range $30-500 \mathrm{~m} / \mathrm{z}$. The constituents were identified by comparing the experimental spectra with spectra from the NIST 98 data bank (NIST/EPA/NISH Mass Spectral Library, version 1.6, U.S.A.) as well as by comparison of their GC Kovats index (Adams, 2007). For quantification purposes, the chromatographic peak areas were determined using, for each compound, the corresponding base ion $(\mathrm{m} / \mathrm{z} 100 \%$ intensity), with the internal standard area as reference.

\subsection{Statistical analysis}

\subsubsection{Analysis of variance}

Analysis of variance (ANOVA) with Type III sums of squares was performed using the GLM (General Linear Model) procedure of the SPSS software, version 21.0 (IBM Corporation, New York, U.S.A.). The fulfillment of the ANOVA requirements, namely the normal distribution of the residuals and the homogeneity of variance, were evaluated using the Kolmogorov-Smirnov with Lilliefors correction (if $n>50$ ) or the Shapiro-Wilk test (if $n<50$ ) and the Levene tests, respectively. All dependent variables were analyzed using one-way ANOVA with or without Welch correction, depending on whether the requirement of the homogeneity of variances was fulfilled or not. The main factor studied was the effect of harvest time on the volatile composition of the three studied olive cultivars. If a statistically significant effect was found, the means were compared using the Tukey honestly significant difference multiple comparison test or the Dunnett T3 test, also depending on whether equal variances could be assumed. All statistical tests were performed at a $5 \%$ significance level.

\subsubsection{Principal component analysis}

Principal component analysis (PCA) was applied to reduce the number of variables in the three olive cultivars to a smaller number of new derived variables (principal component or factors) that adequately summarize the original information, i.e., the effect of collection time and cultivar on the volatile composition of olive leaves and their relation to olive fly cultivar preference. Variables corresponding to 11 of the most abundant volatile compounds identified, olive fly infestation levels, and total volatile amounts $(\mathrm{mg} / \mathrm{kg})$ at the last three sampling dates (4th Oct; 21st Oct, and 9th Nov) were combined. PCA was performed using the SPSS software, version 21.0 (IBM Corporation, New York, U.S.A.).

\section{Acknowledgements}

The authors are grateful to the Portuguese Foundation of Science and Technology for financial support through the project EXCL/AGR-PRO/0591/2012 "Olive crop protection in sustainable production under global climatic changes: linking ecological infrastructures to ecosystem functions". R. Malheiro thanks FCT, POPH-QREN and FSE for PhD grant (SFRH/BD/74675/2010). This manuscript is part of Ricardo Malheiro PhD Thesis. 


\section{References}

Adams, R.P., 2007. Identification of Essential Oil Components by Gaschromatography/Mass Spectrometry, fourth ed. Allured Business Media, Carol Stream, Illinois.

Akacha, N.B., Gargouri, M., 2009. Enzymatic synthesis of green notes with hydroperoxide-lyase from olive leaves and alcohol-dehydrogenase from yeast in liquid/gas reactor. Process Biochem. 44, 1122-1127.

Alagna, F., Kallenbach, M., Pompa, A., De Marchis, F., Rao, R., Baldwin, I.T. Bonaventure, G., Baldoni, L., 2015. Olive fruits infested with olive fly larvae respond with an ethylene burst and the emission of specific volatiles. J. Integr. Plant Biol. http://dx.doi.org/10.1111/jipb.12343.

Aluja, M., Mangan, R.L., 2008. Fruit fly (Diptera: Tephritidae) host status determination: critical conceptual, methodological, and regulatory considerations. Annu. Rev. Entomol. 53, 473-502.

Baccouri, O., Bendini, A., Cerretani, L., Guerfel, M., Baccouri, B., Lercker, G., Zarrouk, M. Ben Miled, D.D. 2008. Comparative study on volatile compounds from Tunisia and Sicilian monovarietal olive oils. Food Chem 111, 322-328.

Biedermann, M., Grob, K., Morchio, G., 1995. On the origin of benzene, toluene ethylbenzene and xylene in extra virgin olive oil. Z. Lebensm. Unters. Forsch. 200, 266-272.

Brahmi, F., Flamini, G., Issaoui, M., Dhibi, M., Dabbou, S., Mastouri, M., Hammami, M., 2012. Chemical composition and biological activities of volatile fractions from three Tunisian cultivars of olive leaves. Med. Chem. Res. 21, 2863-2872.

Burrack, H.J., Zalom, F.G., 2008. Olive fruit fly (Diptera: Tephritidae) ovipositional preference and larval performance in several commercial important olive varieties in California. Ecol. Behav. 101, 750-758.

Campeol, E., Flamini, G., Chericoni, S., Catalano, S., 2001. Volatile compounds from three cultivars of Olea europaea from Italy. J. Agric. Food Chem. 49, 5409-5411.

Campeol, E., Flamini, G., Cioni, P.L., Morelli, I., 2003. Volatile fractions from three cultivars of Olea europaea L. collected in two different seasons. J. Agric. Food Chem. 51, 1994-1999.

Corrado, G., Alagna, F., Rocco, M., Renzone, G., Varricchio, P., Coppola, V., Coppola M., Garonna, A., Baldoni, L., Scaloni, A., Rao, R., 2012. Molecular interactions between the olive and the fruit fly Bactrocera oleae. BMC Plant Biol. 12, 1-17.

Daane, K.M., Jonhson, M.W., 2010. Olive fruit fly: managing an ancient pest in modern times. Annu. Rev. Entomol. 55, 151-169.

De Cristofaro, A., Rotundo, G., Belcari, A., Germinara, G.S., 2007. Effect of age and mating status on the antennal sensitivity of Bactrocera oleae (Rossi) (Diptera Tephritidae) male and female. IOBC/WPRS Bull 30, 23.

Dudareva, N., Negre, F., Nagegowda, D.A., Orlova, I., 2006. Plant volatiles: recent advances and future perspectives. Crit. Rev. Plant Sci. 25, 417-440.

Flamini, G., Cioni, P.L., Morelli, I., 2003. Volatiles from leaves, fruits, and virgin oil from Olea europaea Cv. Olivastra Seggianese from Italy. J. Agric. Food Chem. 51 1382-1386.

Gonçalves, M.F., Malheiro, R., Casal, S., Torres, L., Pereira, J.A., 2012. Influence of fruit traits on oviposition preference of the olive fly, Bactrocera oleae (Rossi) (Diptera: Tephritidae), on three Portuguese olive varieties (Cobrançosa, Madural and Verdeal Transmontana). Sci. Hortic. 145, 127-135.

Guerfel, M., Baccouri, O., Boujnah, D., Zarrouk, M., 2008. Changes in lipid composition, water relations and gas Exchange in leaves of two young 'Chemlali' and 'Chetoui' olive trees in response to water stress. Plant Soil 311, $121-129$.

Heiden, A.C., Kobel, K., Komenda, M., Koppmann, R., Shao, M., Wildt, J., 1999. Toluene emissions from plants. Geophys. Res. Lett. 26, 1283-1286.

Hermoso, M., Uceda, M., Frias, L., Beltrán, G., 2001. Maduración. In: Barranco, D., Fernández-Escobar, R., Rallo, L. (Eds.), El cultivo del olivo. Ediciones MundiPrensa, Madrid, pp. 153-170.

Iannotta, N., Noce, M.E., Ripa, V., Scalercio, S., Vizzarri, V., 2007. Assessment of susceptibility of olive cultivars to the Bactrocera oleae (Gmelin, 1790) and Camarosporium dalmaticum (Thüm.) Zachos \& Tzav.-Klon. attacks in Calabria (Southern Italy). J. Environ. Sci. Health Part B 42, 789-793.
Imperato, A., Corrado, G., Alagna, F., Varricchio, P., Baldoni, L., Rao, R., 2012. Olive molecular response to attack of Bactrocera oleae: identification of up-regulated genes in infested olive fruits. Acta Hortic. 929, 125-128.

Iraqui, R., Vermeulen, C., Benzekri, A., Bouseta, A., Collin, S., 2005. Screening for key odorants in Moroccan green olives by gas-chromatography-olfactometry/aroma extract dilution analysis. J. Agric. Food Chem. 53, 1179-1184.

Kombargi, W.S., Michelakis, S.E., Petrakis, C.A., 1998. Effect of olive surface waxes on oviposition by Bactrocera oleae (Diptera: Tephritidae). J. Econ. Entomol. 91, 993998.

Liscia, A., Angioni, P., Sacchetti, P., Poddighe, S., Granchietti, A., Setzu, M.D., Belcari, A., 2013. Characterization of olfactory sensilla of the olive fly: behavioral and electrophysiological responses to volatile organic compounds from the host plant and bacterial filtrate. J. Insect. Physiol. 50, 705-716.

Malheiro, R., Pinho, P.G., Soares, S., Ferreira, A.C.S., Baptista, P., 2013. Volatile biomarkers for wild mushrooms species discrimination. Food Res. Int. 54, 186 194.

Malheiro, R., Casal, S., Baptista, P., Pereira, J.A., 2015a. A review of Bactrocera oleae (Rossi) impact in olive products: from the tree to the table. Trend. Food Sci. Technol. 44, 226-242.

Malheiro, R., Casal, S., Cunha, S.C., Baptista, P., Pereira, J.A., 2015b. Olive volatiles from Portuguese cultivars Cobrançosa, Madural and Verdeal Transmontana: role in oviposition preference of Bactrocera oleae (Rossi) (Diptera: Tephritidae). Plos One 10, 1-15.

Navrozidis, E., Zartaloudis, Z., Thomidis, T., Karagiannidis, N., Roubos, K., Michailides, Z., 2007. Effect of soil plowing and fertilization on the susceptibility of four olive cultivars to the insect Bactrocera oleae and the fungi Sphaeropsis dalmatica and Spilocaea oleagina. Phytopathology 35, 429-432.

Neuenschwander, P., Michelakis, S., Holloway, P., Berchtold, W., 1985. Factors affecting the susceptibility of fruits of different olive varieties to attack of Dacus oleae (Gmel.) (Dipt., Tephritidae). J. Appl. Entomol. 100, 174-188.

Paré, P.W., Tummlinson, J.H., 1999. Plant volatiles as a defense against insecto herbivores. Plant Physiol. 121, 325-332.

Rizzo, R., Caleca, V., Lombardo, A., 2012. Relation of fruit color, elongation, hardness, and volume to the infestation of olive cultivars by the olive fruit fly, Bactrocera oleae. Entomol. Exp. Appl. 145, 15-22.

Sacchetti, P., Granchietti, A., Landini, S., Vitti, C., Giovannetti, L., Belcari, A., 2008. Relationships between the olive fly and bacteria. J. Appl. Entomol. 132, 682689

Salas, J.J., Sánchez, C., García-González, D.L., Aparicio, R., 2005. Impact of the suppression of lipoxygenase and hydroperoxide lyase on the quality of the green odor in green leaves. J. Agric. Food Chem. 53, 1648-1655.

Scarpati, M.L., Lo Scalzo, R., Vita, G., 1993. Olea europaea volatiles attractive and repellent to the olive fruit fly (Dacus oleae, Gmelin). J. Chem. Ecol. 19, 881-891.

Scarpati, M.L., Lo Scalzo, R., Vita, G., Gambacorta, A., 1996. Chemiotropic behavior of female olive fly (Bactrocera oleae Gmel.) on Olea europaea L. J. Chem. Ecol. 22, 1027-1036.

Sofo, A., Dichio, B., Xiloyannis, C., Masia, A., 2004. Lipoxygenase activity and proline accumulation in leaves and roots of olive tree in response to drought stress. Physiol. Plantarum 121, 58-65.

Spadafora, A., Mazzuca, S., Chiappetta, F.F., Parise, A., Innocenti, A.M., 2008. Oleuropein-specific- $\beta$-glucosidase activity marks the early response of olive fruits (Olea europaea) to mimed insect attack. Agric. Sci. China 7, 703-712.

Tamiru, A., Bruce, J.A.B., Woodcock, C.M., Caulfield, J.C., Midega, C.A.O., Ogol, C.K.P. O., Mayon, P., Birkett, M.A., Pickett, J.A., Khan, Z.R., 2011. Maize landraces recruit egg and larval parasitoids in response to egg deposition by a herbivore. Ecol. Lett. 14, 1075-1083.

Unsicker, S.B., Kunert, G., Gershenson, J., 2009. Protective perfumes: the role of vegetative volatiles in plant defense against herbivores. Curr. Opin. Plant Biol. 12, 479-485.

Wu, J., Baldwin, I.T., 2010. New insights into plant responses to the attack from insect herbivores. Annu. Rev. Genet. 44, 1-24. 